\title{
Frozen Cytology of Meningeal Malignant Solitary Fibrous Tumor/Hemangiopericytoma
}

\author{
Myunghee Kang · Na Rae Kim \\ Dong Hae Chung · Gie-Taek Yie \\ Departments of Pathology and ${ }^{1}$ Neurosurgery, \\ Gil Medical Center, Gachon University College of \\ Medicine, Incheon, Korea \\ Received: January 28, 2019 \\ Revised: February 27, 2019 \\ Accepted: March 20, 2019 \\ Corresponding Author \\ Na Rae Kim, MD \\ Department of Pathology, Gil Medical Center, \\ Gachon University College of Medicine, \\ 21 Namdong-daero 774 beon-gil, Namdong-gu, \\ Incheon 21565, Korea \\ Tel: +82-32-460-3073 \\ Fax: +82-32-460-2394 \\ E-mail: clara_nrk@gilhospital.com
}

\begin{abstract}
A 51-year-old woman presented with severe dizziness. The brain magnetic resonance image revealed a $5.5 \mathrm{~cm}$ multiloculated mass with a thick rim in the left temporal lobe. Cytological examination of frozen diagnosis of the mass showed hypercellular sheets of round and rhabdoid cells in a hemorrhagic background, and two mitotic figures were observed. Histologically, the excised dura-based mass consisted of predominantly round cells with small foci of rhabdoid tumor cells in a pseudoalveolar pattern in a hemorrhagic background, and the cells showed nuclear positivity for signal transducer and activator of transcription 6 as well as frequent mitosis. The mass was diagnosed as a grade 3 solitary fibrous tumor (SFT)/hemangiopericytoma (HPC). The cytological diagnosis of SFT/HPC is challenging because of the heterogeneous cytological findings, such as histological heterogeneity, and because there are no standardized cytological criteria for malignant SFT/HPC. Cytological findings, such as singly scattered small cells, hypercellularity, rare ropy collagen, and round and rhabdoid cells with pseudoalveolar pattern, may assist in the diagnosis of malignant SFT/HPC.
\end{abstract}

Key Words: Solitary fibrous tumors; Hemangiopericytoma; Frozen; Cytology; Central nervous system
A solitary fibrous tumor (SFT)/hemangiopericytoma (HPC) is uncommon and was first described as an angioblastic meningioma. ${ }^{1}$ Currently, this combined term is used to describe a wide pathological spectrum of mesenchymal tumors that share molecular events such as paracentric inversion on chromosome 12q13, resulting in NGFI-A-binding protein 2 (NAB2)/signal transducer and activator of transcription 6 (STAT6) gene fusion with STAT6 nuclear immunopositivity. ${ }^{2}$ Dedifferentiation also occurs in SFT/HPC of the central nervous system (CNS), such as in those of extracranial soft tissue. ${ }^{3}$

Few studies have reported the cytological characteristics of SFT/HPC of the CNS. ${ }^{4}$ Here, we present the cytology of a frozen, histologically-proven, grade 3, meningeal SFT/HPC.

\section{CASE REPORT}

\section{Clinical summary}

A 51-year-old woman visited an emergency center for abrupt onset of severe dizziness, sweating, and vomiting during sleep. The brain diffusion-weighted magnetic resonance image showed a $5.5-\mathrm{cm}$ multiloculated mass with a thick hypointense rim, hemorrhage, and surrounding edema in the left temporal lobe (Fig. 1). Cavernous hemangioma or other brain tumors with hemorrhage were considered, and surgical excision was performed. After complete surgical excision, the patient continued in good health for the next seven months.

\section{Pathological findings}

For diagnosis of the frozen tissue, hemorrhagic and highly vascular crush smears composed of sheets of monotonous round cells with heterochromatic nuclei in a hemorrhagic background were obtained (Fig. 2A). Focal myxoid changes were also found (Fig. 2B). Small round cells were juxtaposed with abundant thin capillaries (Fig. 2C, D). In a background rich in red blood cells, only scant eosinophilic ropy collagen was found juxtaposed to the vessels (Fig. 2E). Small clusters or singly-dispersed roundto-rhabdoid cells with eccentric nuclei and eosinophilic, granular, inclusion-like cytoplasm were found (Fig. 2F). Based on the frozen cytology, the initial differential diagnoses of the frozen tissue were SFT/HPC and meningioma of focal rhabdoid type. Albeit rare, an oncocytic glomus tumor was also considered.

For the permanent diagnosis, formalin-fixed paraffin-embed- 


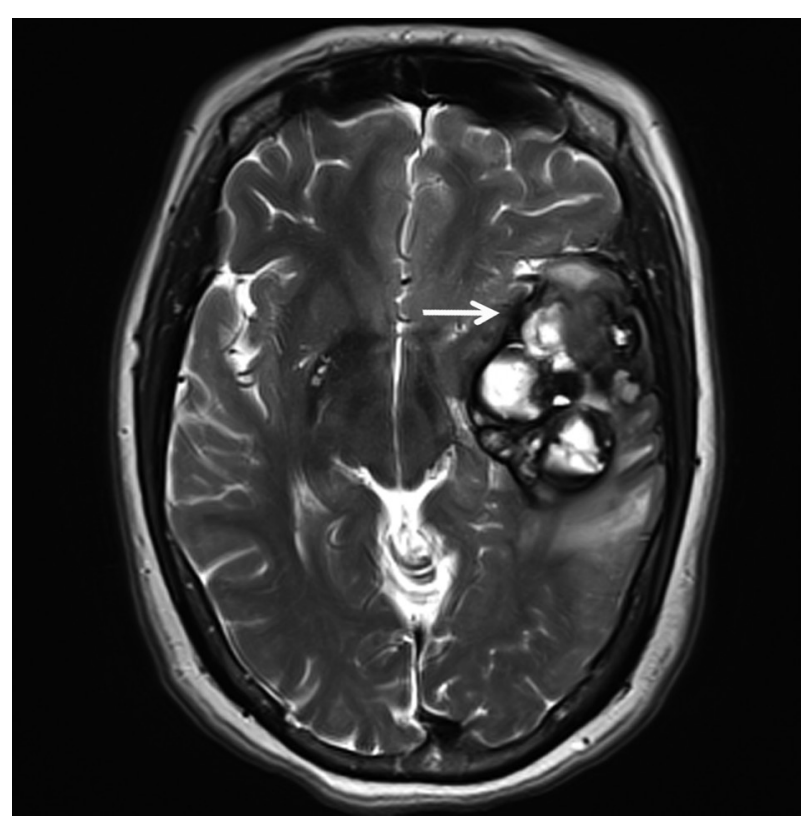

Fig. 1. T2-weighted magnetic resonance imaging reveals a $5.5 \mathrm{~cm}$ multiloculated mass (arrow) with a thick hypointense rim.

ded tissue sections of the entire resected mass were prepared, and they showed a highly vascular and hemorrhagic meningeal mass (Fig. 2G, H) composed of monotonous round cells with irregular nuclei and prominent single nucleoli and a scanty amount of eosinophilic cytoplasm (Fig. 2I). A peripheral solid portion was found, and blood-filled cavities with centrally detached cells, forming a pseudoalveolar and peritheliomatous arrangement, were observed throughout the mass (Fig. 2J). Mitotic activity was 9 mitoses/10 high power fields. Necrosis was not found. The congested portion showed myxoid changes in the loose edematous stroma and loose pseudoalveolar arrangements (Fig. 2K). Focal rhabdoid appearance was also observed, and these cells showed nuclear positivity for INI-1 (1:200, 25/BAF47, BD Bioscience, San Diego, CA, USA) (Fig. 2L, left). Both round and spindle cells showed positive staining for CD34 (1:50, QBEnd 10, Dako, Glostrup, Denmark) and nuclear positivity for STAT6 (1:150, S-20, sc-621, Santa Cruz Biotechnology, Santa Cruz, CA, USA) (Fig. 2L, right). The cells showed negative staining for glial fibrillary acidic antigen (prediluted, polyclonal, Dako), smooth muscle actin (1:100, 1A4, Dako), myogenin (1:50, LO26, Novocastra, Newcastle upon Tyne, UK), desmin (1:100, D33, Dako), epithelial membrane antigen (1:100, E29, Dako), S100 protein (1:600, polyclonal, Dako), synaptophysin (prediluted, DAK-SYNAP, Dako), and c-kit (1:30, T5P5, Novocastra).

Ultrathin sections revealed that the closely packed spindleto-ovoid tumor cells had a moderate amount of cytoplasm con- taining intermediate filaments and lysosomes with a well-formed Golgi apparatus (Fig. 3A). Occasionally, intermediate junctions and pinocytotic vesicles were found. The tumor cells also had cytoplasmic processes containing intermediate filaments (Fig. 3B). Basal lamina-like materials were found around the tumor cells (Fig. 3C). Microvillous processes were found, but paranuclear whorls of intermediate filaments were not observed. Isocitrate dehydrogenase 1 (IDH1) gene R132 mutation was tested on the resected specimen by real time polymerase chain reaction (PCR) using a PNA Clamp IDH1 Mutation Detection Kit (Panagene Ltd., Daejeon, Korea) according to the manufacturer's instructions, and methylation of $\mathrm{O}^{6}$-methylguanine DNA methyltransferase (MGMT) was tested by methylation-specific PCR; IDH1 R132 mutation was not detected, but promoter methylation of MGMT gene was detected. The tumor was diagnosed as a grade 3 SFT/HPC according to a 3-tier system based on the histopathological phenotype and mitotic count by the World Health Organization 2016 CNS tumor classification. ${ }^{4}$

Approval for this case report was obtained from our Institutional Review Board (No. GCIRB 2019-020) with a waiver of informed consent.

\section{DISCUSSION}

In extracranial SFT/HPC cases, a preoperative, cytological, confirmative diagnosis can be established if STAT6 immunostaining of the cell block is performed. ${ }^{2}$ However, cytologic examination of meningeal SFT/HPC cannot be performed until intraoperative frozen smears are made. ${ }^{5}$ Therefore, cytological examination of frozen tissue is important for the correct diagnosis. Cytologic findings of SFT/HPC are nonspecific and include the so-called patternless pattern and various heterogeneous cytologic findings. ${ }^{6}$ SFT/HPC with naked stripped nuclei has been shown to occur outside the CNS, and scattered ropy collagen fragments in the background are the most distinctive cytological findings. ${ }^{6}$ Both intracranial and extracranial SFT/HPC shows scanty to moderate cellular smears of oval-to-spindle cells in a background of irregular ropy fragments of collagen or eosinophilic collagenous matrix. ${ }^{7}$ Most of the cells are dispersed singly or in loose clusters enmeshed in vessels. ${ }^{8}$ Individual tumor cells show uniform bland nuclei with even finely-granular chromatin in lowgrade SFT/HPC. ${ }^{9}$ The most consistent features are the presence of stripped nuclei in the background and thick ropy bands of matrix material. ${ }^{9}$ The predominance of small round cells in the tumor should be used to distinguish SFT/HPC from neurocytoma or paraganglioma. Considering the heterogeneous and wide 

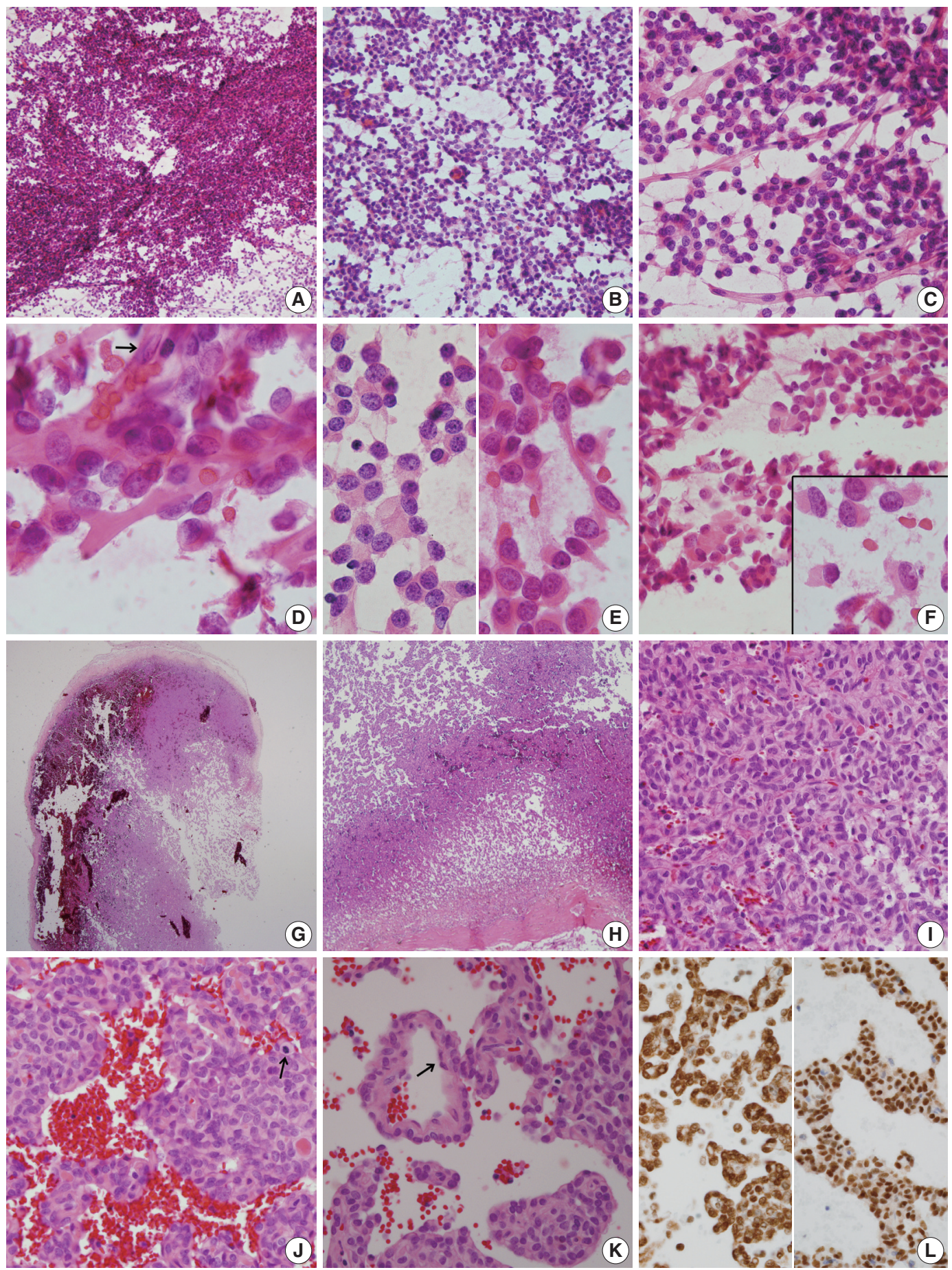

Fig. 2. (A-F) Frozen crush cytology. (A) Cellular smear shows sheets or singly scattered round to oval cells with cellular overlapping. (B) Round- to ovoid-shaped cells have bland nuclear chromatin with a moderate amount of cytoplasm with occasional rhabdoid features. (C) Endothelial cell-lined capillaries crossing the tumor cells. (D) High magnification shows several clusters of oval cells of a pseudoalveolar architecture, coarse chromatin pattern, small inconspicuous nucleoli, and irregular nuclear membranes. Arrow indicates nuclei of endothelial cells. (E) Small round cells (left) and ropy collagenous tails are found. (F) Round to ovoid cells had bland nuclear chromatin with a moderate amount of cytoplasm with occasional rhabdoid features. Inset indicates focal rhabdoid appearance. (G-L) Histological findings. (G, H) Patternless solid growth of spindle to round cells is present with intervening staghorn-like vessels. (I) High magnification shows congested tumor composed of round cells around blood vessels. (J) Pseudoalveolar pattern reveals hypercellularity, pleomorphism, and mitosis (arrow). (K) Congested pseudoalveolar pattern is arranged around blood vessels resembling a pseudorosette-like pattern. Arrow indicates endothelial cells. $(L)$ The tumor cells retain INI-1 immunostainability (left) and nuclear positivity for STAT6 (right). 

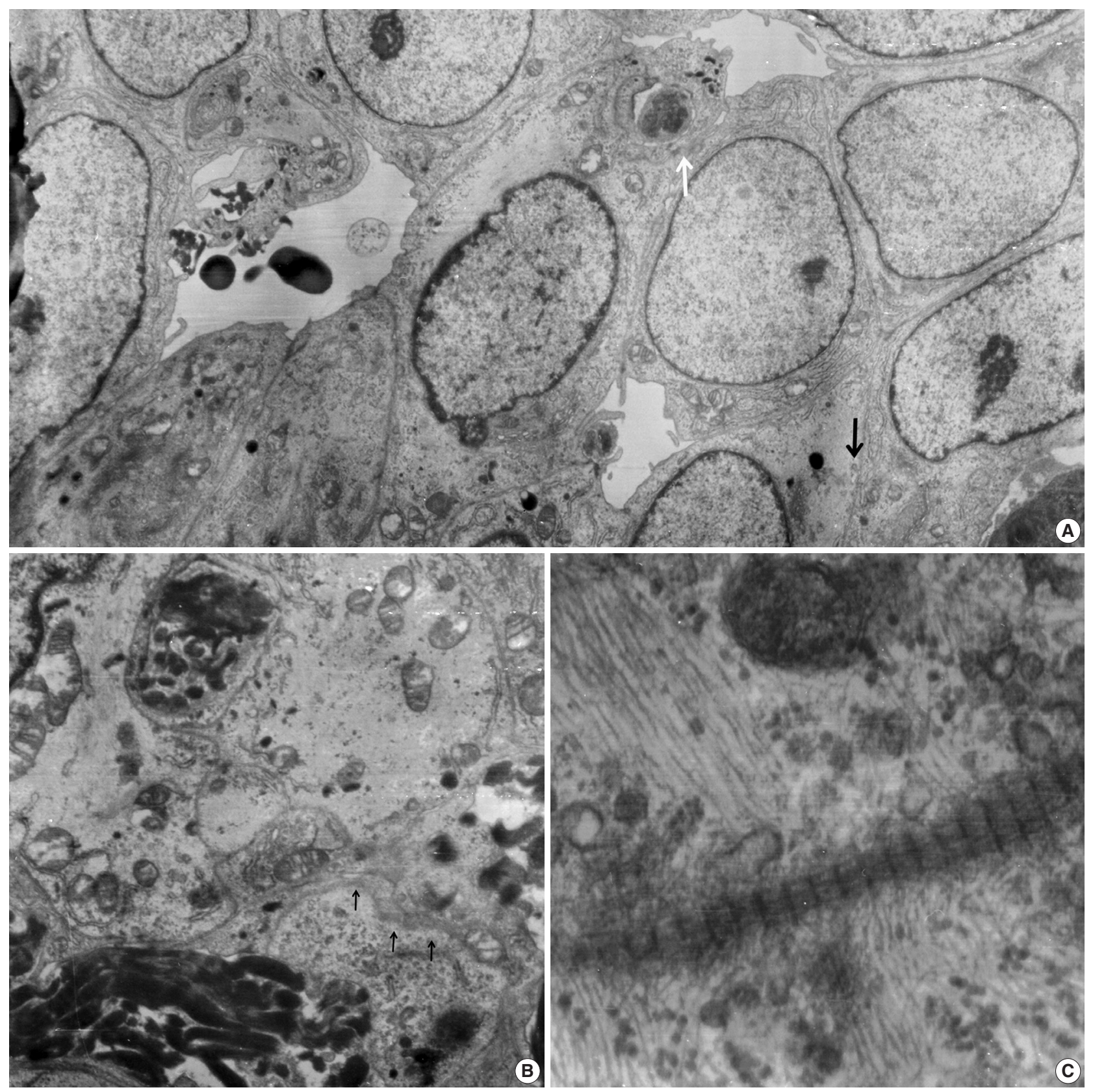

Fig. 3. Electron microscopic findings. (A) Round shaped tumor cells have a moderate amount of cytoplasm filled with intermediate filaments, lysosomes with well-formed Golgi apparatus. Note pinocytotic vesicles (black arrow), intermediate junctions (white arrow) and microvillous processes $(\times 2,500)$. (B) Basal lamina-like materials (arrows) surround the tumor cells filled with intermediate filaments $(\times 5,000)$. (C) Wellformed collagen bundles at the extracellular spaces and cytoplasmic intermediate filaments are found $(\times 3,000)$.

spectrum of histological findings observed in SFT/HPC, cytologic heterogeneity is comprehensible. SFT/HPC of the CNS is a poorly understood mesenchymal neoplasm, ${ }^{4}$ and few reports of intraoperative cytological characteristics have been published. ${ }^{8,10}$ Low to intermediate grade SFT/HPC, i.e., grades 1 and 2, shows cohesive hypercellular sheets of polygonal- to spindle-shaped tumor cells. The tumor cells have a scant amount of wispy cyto- plasm and oval-to-short spindle-shaped nuclei with coarse chromatin, whereas SFT/HPC of higher grade shows increased nuclear pleomorphism with frequent mitoses. ${ }^{4}$ Dense ropy collagen or dilated vascular structures are observed in almost all cases of benign SFT/HPC. ${ }^{2}$ The present case showed scant ropy collagen, which made it difficult to diagnose SFT/HPC using frozen sections. ${ }^{11}$ Despite the standardized cytological data for higher 
grades of SFT/HPC, malignant SFT/HPC shows hypercellularity, pleomorphism, rare ropy collagen, and epithelioid or round cell features with occasional rosette-like structures as well as necrosis and mitosis, similar to high-grade SFT/HPC., ${ }^{911,12}$ Some studies have suggested that a predominance of single cells confirms the presence of high-grade SFT/HPC. ${ }^{11,13}$ Rare cases of extracranial malignant SFT/HPC have shown heterologous mesenchymal differentiation or lipomatous or rhabdomyosarcomatous differentiation. ${ }^{3,14}$ In the present case, rhabdoid-featured cells retained INI-1 and showed negative staining for myogenin or desmin without ultrastructural demonstration of paranuclear whorls of intermediate filaments. In retrospect, the frozen cytology from the present case that showed a scanty amount of background ropy collagen and dispersed predominant round cells with an occasional rhabdoid appearance did not permit a correct frozen diagnosis. In our opinion, these focal rhabdoid features in the high-grade malignant SFT/HPC, which may be a high-grade component, may indicate the initial signs of dedifferentiation from low-grade SFT/HPC.

Besides, branching staghorn vasculature is one of the salient findings of SFT/HPC, and it is not commonly found in frozen cytology but instead in the cell block. Thus, the vascular pattern does not contribute to the frozen cytologic diagnosis of SFT/ HPC. ${ }^{12}$ Spindle cell predominant smears in SFT/HPC must be distinguished from nerve sheath tumors and fibrous histiocytoma. Meningeal SFT/HPC may show dispersed small monomorphous cells. Smears from a benign peripheral nerve-sheath tumor are composed of end-tapering spindle cells arranged in interlacing bundles in a myxoid background, and wavy nuclear buckling and thick hyalinized vessels may be found. In the present case, the frozen sections showed a myxoid edematous background, but this myxoid background is uncommon in the cytology of SFT/HPC with neither spindle cell nor round cell morphology. ${ }^{12}$ A predominant spindle cell morphology and scattered background amorphous wispy magenta materials in a hemorrhagic background cause problems during diagnosis. A rarely-observed, monotonous, round cell-predominant SFT/HPC, like in the present case, should be distinguished from neurocytoma, paraganglioma, glomus tumor, and glomangiopericytoma, which are uncommon meningeal tumors. ${ }^{15} \mathrm{~A}$ peritheliomatous or pseudoalveolar arrangement in cystic changes, which is unusual for SFT/HPC, is commonly found in glomus tumor/glomangiopericytoma or synovial sarcoma. ${ }^{16}$ Capillaries crossing related to tumor cells as well as hemorrhagic cytological smears are shared cytological findings for glomus tumor, paraganglioma, and SFT/ HPC. When compared with the knobby oval- to spindle-shaped tumor cells of SFT/HPC, the cytology of glomus tumor or glomangiopericytoma is characterized by a fine vasculature surrounded by monotonous round cells with punched out nuclei and amphophilic cytoplasm with an indistinct cell border. Paraganglioma cytology also shows round cells with moderate nuclear pleomorphism and fine granular cytoplasm enveloped by thin vessels. ${ }^{17}$

It is important to differentiate SFT/HPC from meningioma. The indistinct cell border and stippled chromatin of meningioma differ from the cytology observed in the present case. Intraoperative cytologic findings of meningiomas have been well-described: nuclear grooves, intranuclear inclusions, and psammoma bodies as well as abundant wispy cytoplasm. However, similar to the present case, a predominance of epithelioid cells and small cell change or rhabdoid cells can be found in higher grade meningiomas. Rhabdoid meningioma shows eccentrically placed vesicular nuclei with eosinophilic plump hyaline cytoplasm and short broad processes. ${ }^{18}$ The nuclei have occasional nuclear inclusions with no nuclear grooves and whorls. Oncocytic glomus tumor can be distinguished with the histological findings. Other CNS neoplasms showing rhabdoid cells are astrocytomas, glioblastomas, ependymomas and atypical teratoid/rhabdoid tumors, CNS embryonal tumors with rhabdoid features, choroid plexus carcinomas, sarcomas, and germ cell tumors. ${ }^{19}$

The present case showed MGMT methylation. Although a clear correlation between MGMT methylation status and response has not yet been clarified in SFT/HPC (unlike glioblastoma), SFT/HPC with or without MGMT methylation enhanced the response to alkylating agents by inhibiting DNA repair in a previous study using temozolomide or dacarbazine. ${ }^{20}$

The present tumor was diagnosed as grade $3 \mathrm{SFT} / \mathrm{HPC}$, although the frozen smears did not show the typical cytological findings of SFT/HPC such as a paucity of collagenous stroma and rhabdoid cells masquerading as high grade meningioma, as well as a pseudoalveolar pattern, which are heterogeneous.

\section{ORCID}

Myunghee Kang: https://orcid.org/0000-0003-4083-888X

Na Rae Kim: https://orcid.org/0000-0003-2793-6856

Dong Hae Chung: https://orcid.org/0000-0002-4538-0989

Gie-Taek Yie: https://orcid.org/0000-0002-8706-7253

\section{Author Contributions}

Conceptualization: NRK.

Data curation: DHC. 
Investigation: GTY.

Writing—original draft: MK, NRK.

Writing_review \& editing: NRK, DHC.

\section{Conflicts of Interest}

The authors declare that they have no potential conflicts of interest.

\section{REFERENCES}

1. Bailey P, Cushing H, Eisenchardt L. Angioblastic meningioma. Arch Pathol Lab Med 1928; 6: 953-90.

2. Tani E, Wejde J, Åström K, Wingmo IL, Larsson O, Haglund F. FNA cytology of solitary fibrous tumors and the diagnostic value of STAT6 immunocytochemistry. Cancer Cytopathol 2018; 126: 36-43.

3. Maekawa A, Kohashi K, Yamada Y, et al. A case of intracranial solitary fibrous tumor/hemangiopericytoma with dedifferentiated component. Neuropathology 2015; 35: 260-5.

4. Giannini G, Rushing EJ, Hainfellner JA, et al. Solitary fibrous tumour/haemangiopericytoma. In: Louis DN, Ohgaki H, Wiestler OD, Cavenee WK, eds. WHO classification of tumours of the central nervous system. 4th ed. Lyon: IARC, 2016; 249-54.

5. Samal S, Kalra R, Sharma J, Singh I, Panda D, Ralli M. Comparison between crush/squash cytology and frozen section preparation in intraoperative diagnosis of central nervous system lesions. Oncol J India 2017; 1: 25-30.

6. Tihan T, Viglione M, Rosenblum MK, Olivi A, Burger PC. Solitary fibrous tumors in the central nervous system: a clinicopathologic review of 18 cases and comparison to meningeal hemangiopericytomas. Arch Pathol Lab Med 2003; 127: 432-9.

7. Clayton AC, Salomao DR, Keeney GL, Nascimento AG. Solitary fibrous tumor: a study of cytologic features of six cases diagnosed by fine-needle aspiration. Diagn Cytopathol 2001; 25: 172-6.

8. Gill SS, Bharadwaj R. Cytomorphologic findings of hemangiopericytoma of the meninges: a case report. Indian J Pathol Microbiol 2007; 50: 422-5.

9. Baliga M, Flowers R, Heard K, Siddiqi A, Akhtar I. Solitary fibrous tumor of the lung: a case report with a study of the aspiration biopsy, histopathology, immunohistochemistry, and autopsy findings. Diagn Cytopathol 2007; 35: 239-44.

10. Sandoh K, Ishida M, Okano K, et al. Cytological characteristics of meningeal solitary fibrous tumor metastatic to the lung: a case report with immunocytochemical analysis. Mol Clin Oncol 2018; 9: 17-20.

11. Khanchel F, Driss M, Mrad K, Romdhane KB. Malignant solitary fibrous tumor in the extremity: cytopathologic findings. J Cytol 2012; 29: 139-41.

12. Bishop JA, Rekhtman N, Chun J, Wakely PE Jr, Ali SZ. Malignant solitary fibrous tumor: cytopathologic findings and differential diagnosis. Cancer Cytopathol 2010; 118: 83-9.

13. Ali SZ, Hoon V, Hoda S, Heelan R, Zakowski MF. Solitary fibrous tumor: a cytologic-histologic study with clinical, radiologic, and immunohistochemical correlations. Cancer 1997; 81: 116-21.

14. Kwon JH, Song JS, Jung HW, Lee JS, Cho KJ. Malignant solitary fibrous tumor with heterologous rhabdomyosarcomatous differentiation: a case report. J Pathol Transl Med 2017; 51: 171-5.

15. Deb P, Kinra P, Bhatoe HS. Intraoperative cytology of central neurocytoma mimicking oligodendroglioma. J Cytol 2011; 28: 219-22.

16. Shetty KJ, Rao C, Prasad HL. Glomangiopericytoma versus solitary fibrous tumor: an omental tumor with unusual diagnostic dilemma. Indian J Surg Oncol 2016; 7: 475-8.

17. Naniwadekar MR, Jagtap SV, Kshirsagar AY, Shinagare SA, Tata HR, Sahoo K. Fine needle aspiration diagnosis of carotid body tumor in a case of multiple paragangliomas presenting with facial palsy: a case report. Acta Cytol 2010; 54: 635-9.

18. Xiao GQ, Burstein DE. Cytologic findings of rhabdoid meningioma in cerebrospinal fluid. Acta Cytol 2008; 52: 118-9.

19. Louis DN, Perry A, Reifenberger G, et al. The 2016 World Health Organization Classification of tumors of the central nervous system: a summary. Acta Neuropathol 2016; 131: 803-20.

20. Stacchiotti S, Tortoreto M, Bozzi F, et al. Dacarbazine in solitary fibrous tumor: a case series analysis and preclinical evidence vis-avis temozolomide and antiangiogenics. Clin Cancer Res 2013; 19: 5192-201. 